\title{
A Case of Obstructing Candida Glabrata Bladder Mucus Treated with Intravesicular Amphotericin-B
}

\author{
Dr. Glenn Goodwin ${ }^{* 1}$, D.O., Dr. Nicholas Gaeto ${ }^{2}$, D.O., Mara Seat ${ }^{3}$, OMS III \\ ${ }^{1}$ Aventura Medical Center and Hospital; 20900 Biscayne Blvd.; Miami, FL 33180 \\ ${ }^{2}$ Einstein Medical Center; 5401 Old York Road, Suite 363; Philadelphia, PA 19141 \\ ${ }^{3}$ Nova Southeastern University School of Osteopathic Medicine; 3200 S University Dr, Davie, FL 33328 \\ *Corresponding author: Dr. Glenn Goodwin, PGY 2, D.O.; ggoodwin954 @ gmail.com
}

Received 21 September 2021;

Accepted 30 September 2021;

Published 09 October 2021

\begin{abstract}
Summary
A 51-year-old female with a past medical history of poorly controlled diabetes mellitus presented to the emergency department with signs of emphysematous cystitis and pyelonephritis with associated Enterococcus faecalis bacteremia and Candida Glabrata (C.Glabrata) fungemia. She was treated with micafungin and amoxicillin with improvement in her symptoms. During her course she underwent repeat imaging which showed improvement in her cystitis and no obstruction in her bladder. She was discharged on home IV micafungin. She returned to the emergency department the next day with acute urinary obstruction and was found to have a completely obstructing mucus ball in her bladder. This was removed cystoscopically and surgical cultures grew C. glabrata. She was successfully treated with intra-vesicular amphotericin B and was discharged.
\end{abstract}

\section{Background}

Candida glabrata genitourinary infections present treatment difficulties. Due to azole resistance, therapeutic options are limited to echinocandins and amphotericin B. Echinocandins are poorly concentrated in the urine and the evidence for their use in bladder infections is mixed and based on case reports. Amphotericin B is highly effective but has significant toxicities which can limit its use.

A rare complication of fungal cystitis is the formation of fungal balls in the kidneys or bladder. Often associated with candiduria, fungal balls can cause obstruction leading to urosepsis or other complications. They most often require surgical removal. To our knowledge, there are no cases of obstructing mucus associated with fungal cystitis in the literature.

We present this case to highlight a treatment failure of micafungin in the treatment of fungal cystitis, associated with the rapid development of obstructing fungal mucus requiring surgical treatment. We highlight the importance of considering fungal balls or mucus in the case of a patient who continues to have urinary symptoms despite treatment.

\section{Case Presentation}

Our patient is a 50-year-old female who presented to the emergency department with acute abdominal pain and urinary retention. She had a medical history significant for poorly controlled type 2 diabetes mellitus with a hemoglobin a1c greater than $14 \%$, as well as coronary artery disease. She had recently been discharged from an inpatient service for an E. Coli bacteremia from a urinary source that was complicated by obstruction. At the time she had a urinary catheter placed that had since been discontinued. She had successfully completed her outpatient course of cefalexin. Approximately a week later she returned with worsening symptoms and a recurrence of her acute urinary retention.

\section{Objective Findings}

The patient was evaluated in the emergency department. She was afebrile, with unremarkable vital signs significant only for a borderline tachycardia of $98 \mathrm{bpm}$. Her physical exam revealed a normal cardiac and pulmonary exam, no rash or neurologic deficits, and a firm, distended abdomen that was diffusely tender. She had significant metabolic derangements on laboratory studies, including a blood glucose level of $1325 \mathrm{mg} / \mathrm{dl}$, creatinine of $1.96 \mathrm{mg} / \mathrm{dL}$ from a baseline of $0.5 \mathrm{mg} / \mathrm{dl}$, with a Blood Urea Nitrogen of $33 \mathrm{mg} / \mathrm{dL}$, and a serum bicarbonate of $14 \mathrm{mEq} / \mathrm{L}$ with a normal anion gap of $11 \mathrm{mEq} / \mathrm{L}$. Additionally, her sodium was 
$107 \mathrm{mEq} / \mathrm{L}$, potassium $5.2 \mathrm{mmol} / \mathrm{L}$, chloride $81 \mathrm{mEq} / \mathrm{L}$, magnesium of $2.7 \mathrm{mg} / \mathrm{dL}$. Her lipase and troponin were within reference limits, as was a beta-hydroxybutyrate. Her complete blood count showed a white count of $4.71(109 / \mathrm{L})$ with $68.7 \%$ segmented neutrophils and $15 \%$ bands, $13.1 \%$ lymphocytes, $1 \%$ monocytes, and 0 eosinophils or basophils. She had a normocytic anemia which was noted on previous studies. A urine drug screen was negative for all tested substances. Her urinalysis was significant for $>1000 \mathrm{mg} / \mathrm{dL}$ glucose, 100mg protein, >20 Red Blood Cells and White Blood Cells, positive leukocyte esterase, moderate bacteria, and negative ketones.

She underwent computed tomography (CT) cross sectional imaging of her abdomen and pelvis which was significant for severe emphysematous cystitis and bilateral emphysematous pyelonephritis, bilateral hydroureteronephrosis, and a concern for pneumoperitoneum.

\section{Clinical Course}

She was admitted to the inpatient medicine service for management of her hyperglycemia and pyelonephritis. She was evaluated by the urology service and was found to not require urgent surgery. She underwent placement of a urinary catheter for bladder decompression, and was treated with intravenous insulin, normal saline, and electrolyte repletion. She was also treated with cefepime and vancomycin empirically.

On day 2 of admission, her blood cultures became positive for yeast and she was started on micafungin. Other blood cultures became positive for enterococcus faecalis without resistance and her antibiotics were de-escalated to amoxicillin. Her blood cultures were speciated to candida glabrata. Her initial urine cultures had no growth, but a repeat sample three days after admission grew $\mathrm{C}$. glabrata.

Due to concern of the toxicity of amphotericin B, the patient was maintained on micafungin for her candidemia. She was evaluated by ophthalmology due to her candidemia and had no findings of candida endophthalmitis.

The patient underwent follow up imaging of her bladder to rule out perforation including a CT cystogram which showed continued signs of emphysematous cystitis without bladder rupture. During this procedure, she suffered acute urinary retention despite her urinary catheter placement, requiring multiple catheter exchanges over the next 48 hours. Her urine was grossly significant for small amount of obstructing tissue.

The patient continued to improve and was discharged home on a course of intravenous (IV) micafungin and with a urinary catheter in place on day eight of admission with a diagnosis of acute candidal pyelonephritis.

The next day the patient returned to the emergency department with acute abdominal pain after her home care nurse was unable to flush her catheter. At the time her laboratory studies were significant for a new acute renal injury and hyperglycemia. A point of care ultrasound performed in the emergency department was concerning for a mass versus sediment in the lumen of the bladder, with concern for rupture and prolapse of the intestines into the bladder. The patient was taken urgently to the operating room by the urology service for cystoscopy.

\section{Images}

\section{Initial CT:}

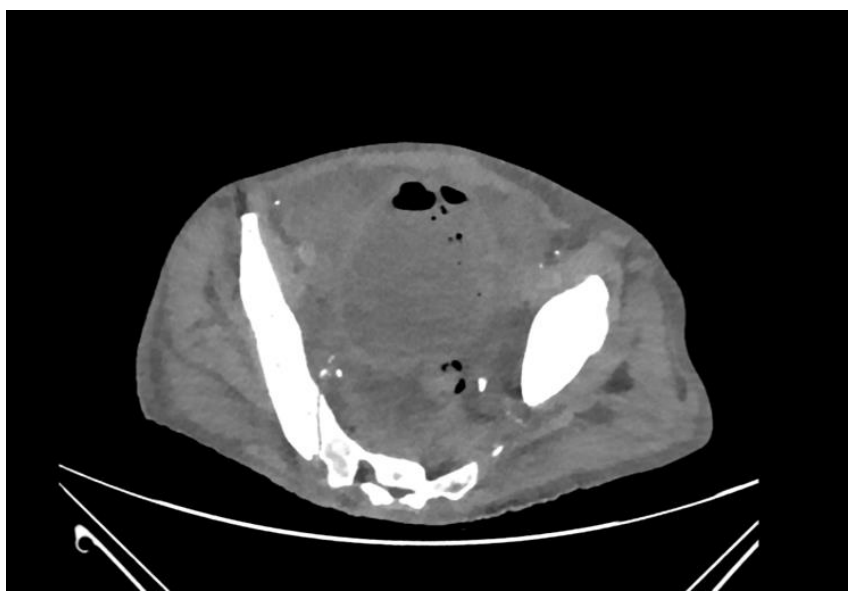

\section{CT cystogram:}

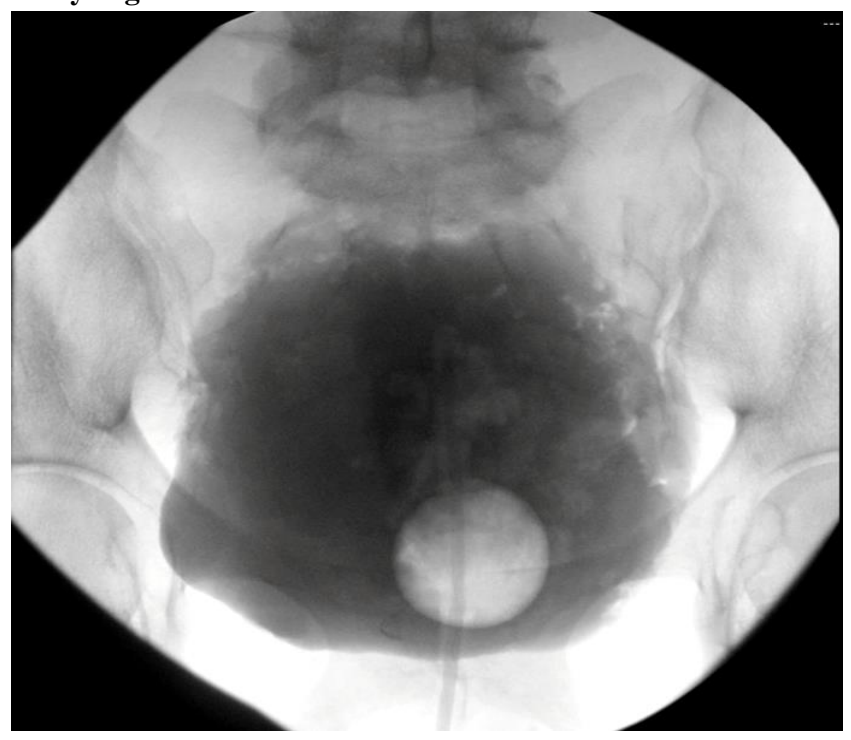

Gross surgical specimen:

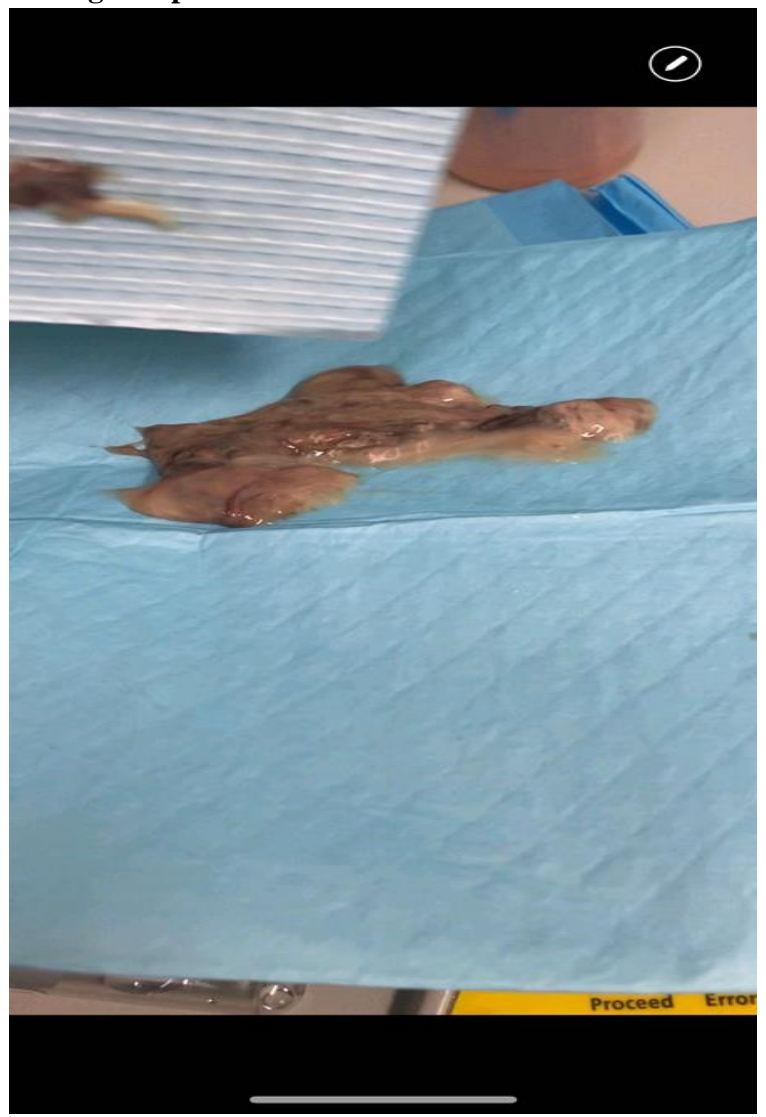




\section{Treatment}

In the operating room, the patient's entire bladder was found to be filled with an obstructing mucus preventing visualization. The bladder was aggressively irrigated and copious amounts of mucus was removed. Further examination with the resectoscope showed significant bladder wall necrosis, other areas of significant mucus collection, but no rupture of the bladder. The remainder of the mucus was removed, and the patient was readmitted to the medical service.

Due to the concern of low micafungin excretion in the urine, the patient was treated with intravesicular amphotericin B 150mg in $3,000 \mathrm{~mL}$ sterile water in the evening for two days, which was infused through the urinary catheter. Her micafungin was continued through the end of her two-week course. Surgical cultures in the operating room continued to grow $\mathrm{C}$. glabrata.

\section{Outcome and Follow-Up}

The patient continued to show improvement. On day five of her second hospitalization she was discharged home with continued home care services and has not had recurrence of her candidal cystitis as of several weeks post discharge.

\section{Discussion}

There are few investigations of C. Glabrata compared to other candida species and C. Glabrata is associated with a high mortality rate compared to other species. Very little is known about the infectivity. There are only two established animal models of experimental C. Glabrata infections (systemic and vaginal) ${ }^{[1]}$.

One study by Fidel et al examined systemic vs vaginal $\mathrm{C}$. Glabrata infections by introducing several isolates of C. Glabrata to immunosuppressed mice with Fluorouracil $(150 \mathrm{mg} / \mathrm{kg}) \mathrm{IV}$ or gamma radiation with 450 to 550 rads to achieve infection ${ }^{[2]}$. The smallest inoculum required to infect was $10^{8}$ blastoconidia. The lack of lethality in the study does not match the mortality in C. Glabrata infections in humans, however, the clinical experience is advanced state of debilitation in humans ${ }^{[2]}$. Regarding treatment, Amphotericin was most efficacious while fluconazole was generally ineffective ${ }^{[2]}$. Moreover, a lack of correlation between in vitro susceptibility tests and in vivo efficacy was often evident ${ }^{[2]}$.

\section{Key Features of Candida Glabrada}

C. glabrata is the only Candida species that does not form pseudohyphae at temperatures above $37^{\circ} \mathrm{C}^{[1]}$.

It can be found as a part of your natural microflora, even existing in the GI tract, the mouth, and the genital area. It has a high resistance to antifungal medications which makes it difficult to treat ${ }^{[3]}$.

It is most likely to infect the urinary tract, genitals, mouth or bloodstream. C. glabrata causes typical UTI symptoms such as urgency, frequency, burning in urination, blood in the urine and pelvic or abdominal pain ${ }^{[3]}$.

Candida glabrata is the second most prevalent yeast pathogen in humans after Candida albicans ${ }^{[5]}$.

The incidence of C. glabrata is higher in adults than in children, and lower in neonates ${ }^{[6]}$.

Unlike candida albicans, c glabrata is not polymorphic and only grows as blastoconidia (yeast). It ferments only glucose and trehalose ${ }^{[6]}$.
They have an ability to grow and divide after being engulfed by macrophages, making them extremely difficult to treat ${ }^{[7]}$.

For many years, C. Glabrata was considered relatively nonpathogenic saprophyte of normal flora and not associated with serious infection in humans ${ }^{[5]}$. Post use of immunosuppressants and broad spectrum antibiotics, mucosal and systemic infections from C. Glabrata has increased significantly ${ }^{[6]}$.

- Compared to other Candida Albicans (NCAC) species, C. glabrata has the highest mortality rate ${ }^{[6]}$. Year after year, these pathological circumstances are more recurrent and problematic to treat, especially when patients reveal any level of immunosuppression. These difficulties arise from the capacity of C. Glabrata to form biofilms in addition to its high resistance to traditional antifungal therapies ${ }^{[6]}$.

- Little is known about the hospital reservoirs of C. Glabrata, however, hands and surfaces are suggested.

Gene EPA6 expression increases during in-vivo UTI, suggesting C. Glabrata can adapt to different environmental conditions ${ }^{[6]}$.

\section{Risk Factors for C. Glabrata infection and fungus balls}

Like most fungal infections, patients suffering from diabetes mellitus are most at risk, followed by renal transplant, extremes of age, female, sex, concomitant bacteriuria, instrumentation of the urinary tract, prolonged hospitalization, broad spectrum antibiotics, bladder dysfunction, urinary stasis, nephrolithiasis, Intensive Care Unit (ICU) admission, congenital abnormalities of the urinary tract ${ }^{[6]}$.

Additional risk factors are those who wear dentures and on antibiotics for prolonged period ${ }^{[6]}$.

Fungal balls or fungal bezoars are rare pathological complications almost only exclusively seen in an immunocompromised host with candiduria.

\section{Treatment}

Therapy for fungal balls/bezoars with either fluconazole (200 to $400 \mathrm{mg}$ [3 to $6 \mathrm{mg} / \mathrm{kg}$ ] orally once daily) or amphotericin B deoxycholate ( 0.3 to $0.6 \mathrm{mg} / \mathrm{kg}$ IV daily) with or without flucytosine (100 mg orally per day divided into four doses). This should be continued until a surgical or endoscopic procedure to remove the fungus ball has been accomplished, symptoms have resolved, and urine cultures are negative ${ }^{[1]}$.

Fluconazole is the antifungal agent of choice for fluconazolesusceptible species of candida, achieving high urine concentrations with the oral formulation ${ }^{[8]}$. Echinocandins are generally not recommended because of their inability to adequately penetrate the urine ${ }^{[9]}$. In the unusual patient who has C. Krusei or fluconazoleresistant C. Glabrata cystitis, Amphotericin B bladder irrigation can sometimes prove useful with or without flucytosine ${ }^{[9]}$. Candida glabrata may be intermediately resistant to all azoles and about $20 \%$ of strains develop resistance during therapy and prophylaxis with fluconazole ${ }^{[6]}$. Instead, bladder irrigation with amphotericin B Deoxycholate for 5 days is suggested ${ }^{[6]}$. The combination of multi-route antifungal therapy (amphotericin and flucytosine) and decompression of the collecting system was successful and avoided the need for surgical source control of the C. glabrata bezoars ${ }^{[10-12]}$. 
Studies in experimental animals and humans have shown that Amphotericin B persists in urine for days to weeks after a single IV dose in levels that should inhibit candidal organisms and thereby obviate the need for frequent dosing ${ }^{[8]}$.

\section{Why does Micafungin not penetrate the urine?}

A case report from Marcelin shows Micafungin use from $50 \mathrm{mg}$ to $200 \mathrm{mg}$ daily from 2 weeks to 25 days demonstrated some benefit

${ }^{[3]}$. Micafungin has little to no glomerular filtration and tubular secretion, but can make its way in small doses unchanged ${ }^{[3]}$. Even with only $1 \%$ of the drug reaching the urine, about $0.06 \mu \mathrm{g} / \mathrm{mL}$ of micafungin in the urine could exceed the minimum inhibitory concentration (MIC) needed to treat Candida Glabrata, which is usually very low (MIC $\leq 0.015$ to $0.5 \mu \mathrm{g} / \mathrm{mL}$ ) ${ }^{[3]}$. Although micafungin does not penetrate extensively into the urine, it has been shown in animal models a wide distribution in many organs and tissues, including kidneys ${ }^{[10]}$. Because very little of the drug is actually needed to treat C. Glabrata, Micofungin could be considered as a reasonable starting therapy but certainly not the best. If the clinician decides to start with Micafungin, the threshold for changing therapies should be quite low.

\section{Learning Points/Take Home Messages}

1. C. Glabrada is a rare but lethal cause of urinary tract infections

2. Those most at risk are the immunocompromised, diabetic, and long-term use antibiotics patients.

3. Fungus balls are a rare complication of this infection, requiring urological intervention and antigungal treatments

4. A good initial therapy for C. Glabrada infection is oral Fluconazole or Amphotericin B +/- Flucytosine. If fungus balls are present, continue this therapy until the balls are cystoscopically removed and the patient has resolution of symptoms. The Amphotericin treatment can initially begin as IV but can be switched to intravesicular pending clinical course.

5. C. Glabrada and other previously rare fungal infections are unfortunately on the rise and complication rate hinges on swift diagnostic accuracy and treatment.

\section{List of abbreviations}

C. Glabrada (Candida Glabrada)

Computed Tomography (CT) scan

Intravenous (IV)

Minimum Inhibitory Concentration (MIC)

\section{Data Availability}

Data for C. Glabrata mucus balls are very limited but can be found by utilizing the references listed below as well as the "Up to Date" online resource.

\section{Conflicts of Interest}

The authors declare that there is no conflict of interest regarding the publication of this paper.

\section{Funding Statement}

There was no funding needed or utilized in the construction of this paper.

\section{Authors' contributions}

NG was responsible for describing the case trajectory and providing the original images. MS contributed to the data collection regarding the various treatment options for C. Glabrata. GG compiled the information and formulated the final paper as well as acting as the primary corresponding author. All authors read and approved the final manuscript.

\section{Acknowledgments}

This paper was made with the help and support of Albert Einstein Medical Center as well as Aventura Hospital and Medical Center. The views expressed do not necessarily reflect those of the aforementioned hospitals.

\section{References}

[1] Cox, Gary M., "Candida Infections of the Bladder and Kidneys." UpToDate, www.uptodate.com/contents/candida-infections-of-thebladder-and-kidneys?_escaped_fragment_=.

[2] Fidel PL Jr, Vazquez JA, Sobel JD. Candida glabrata: review of epidemiology, pathogenesis, and clinical disease with comparison to C. albicans. Clin Microbiol Rev. 1999;12(1):80-96. doi:10.1128/CMR.12.1.80

[3] "Marcelin, J., Pharm to Exam Table - Candida Glabrata Urinary Tract Infections." Division of Infectious Diseases, 29 Apr. 2019, blog.unmc.edu/infectiousdisease/2018/12/21/pharm-to-exam-table-candidaglabrata-urinary-tract-infections/.

[4] Pappas PG, Rotstein CMF, Betts RF, et al. Micafungin versus Caspofungin for Treatment of Candidemia and Other Forms of Invasive Candidiasis. Clinical Infectious Diseases. 2007;45:883-893.

[5] Rupinder Kaur, Renee Domergue, Margaret L Zupancic, Brendan P Cormack,A yeast by any other name: Candida glabrata and its interaction with the host, Current Opinion in Microbiology, Volume 8, Issue 4, 2005, Pages 378-384, ISSN 1369-5274, https://www.sciencedirect.com/science/article/pii/S13695 27405000871

[6] Sónia Silva, Melyssa Negri, Mariana Henriques, Rosário Oliveira, David W. Williams, Joana Azeredo, Candida glabrata, Candida parapsilosis and Candida tropicalis: biology, epidemiology, pathogenicity and antifungal resistance, FEMS Microbiology Reviews, Volume 36, Issue 2, March 2012, Pages 288-305, https://doi.org/10.1111/j.1574-6976.2011.00278.x

[7] Palazzi, Debra L., Acute infectious cystitis: Management and prognosis in children older than two years and adolescents

[8] Fisher JF, Woeltje K, Espinel-Ingroff A, Stanfield J, DiPiro JT. Efficacy of a single intravenous dose of amphotericin B for Candida urinary tract infections: further favorable experience. Clin Microbiol Infect. 2003 Oct;9(10):1024-7. doi: 10.1046/j.14690691.2003.00711.x. PMID: 14616745.

[9] Malani AN, Kauffman CA. Candida urinary tract infections: treatment options. Expert Rev Anti Infect 
Ther. $2007 \quad$ Apr;5(2):277-84. doi: 10.1586/14787210.5.2.277. PMID: 17402842.

[10] Pieralli F, Bazzini C, Vannucchi V, Mancini A, Nozzoli C. A case of Candida glabrata severe urinary sepsis successfully treated with micafungin. Med Mycol Case Rep. $2014 \quad$ May 10;5:1-3. doi: 10.1016/j.mmcr.2014.04.003. PMID: 24936401; PMCID: PMC4052353.

[11] Rohloff MA, Shakuri-Rad J, Dehaan AP (2017) Candida bezoars in adults: determining optimal management, Journal of Endourology Case Reports 3:1, 45-48, DOI: 10.1089/cren.2017.0021.

[12] Viswambaram, P., Misko, J., Rawlins, M., Clark, S., Dyer, J., \& Hayne, D. (2020). Multi-route antifungal administration in the management of urinary Candida glabrata bezoar. Urology case reports, 33, 101275. https://doi.org/10.1016/j.eucr.2020.101275 cc) (i)

Open Access This article is licensed under a Creative Commons Attribution 4.0 International License, which permits use, sharing, adaptation, distribution and reproduction in any medium or format, as long as you give appropriate credit to the original author(s) and the source, provide a link to the Creative Commons license, and indicate if changes were made. The images or other third party material in this article are included in the article's Creative Commons license, unless indicated otherwise in a credit line to the material. If material is not included in the article's Creative Commons license and your intended use is not permitted by statutory regulation or exceeds the permitted use, you will need to obtain permission directly from the copyright holder. To view a copy of this license, visit https://creativecommons.org/licenses/by/4.0/.

(c) The Author(s) 2021 